\title{
Frauen in der Wissenschaft - Frauen und \\ Wissenschaft. Liberale und radikale \\ Perspektiven in einem globalen Rahmen
}

Francisco O. Ramirez

Der größte Teil der geschlechtersoziologischen Literatur beschäftigt sich mit der Thematik der fortdauernden Ungleichheit zwischen Frauen und Männern. Die These der Persistenz von Ungleichheit wird mit verschiedenen interaktions- und organisationstheoretischen sowie sozialstrukturellen Ansätzen gestützt, die ihrerseits im Forschungsdesign und in der Methode beträchtliche Unterschiede aufweisen. Eine so starke Übereinstimmung könnte darauf hinweisen, daß sich in bezug auf die Ungleichheit zwischen den Geschlechtern wenig verändert hat. Doch gibt es gleichzeitig viele Hinweise darauf, daß im Gegenteil Frauen und Männer in den verschiedenen Bereichen zunehmend gleichgestellt sind. Der Beitrag greift dieses Paradox auf und diskutiert die folgende These: Der historische Siegeszug egalitärer Standards führt weltweit zu faktischen Zugewinnen an Gleichheit und trägt ebenfalls zur Verbreitung von Gleichheitserwartungen bei. Diese Erwartungen erhöhen wiederum die Wahrscheinlichkeit, daß fortbestehende Unterschiede überhaupt wahrgenommen werden und der Grad dieser Wahrnehmung ausgedehnt wird. Schließlich liefern egalitäre Erwartungen Interpretationsmodelle, um geschlechtsbezogene Ungleichheiten überhaupt als grundlegende Ungerechtigkeit klassifizieren zu können. Selbstverständlich gilt diese These für alle Formen der Ungleichheit in der jetzigen Welt. Die breitere Diskussion über Ungleichheit trägt wiederum dazu bei, geschlechtsbezogene Ungleichheit wahrzunehmen wie auch umgekehrt. Dieser Beitrag beschränkt sich auf die Ungleichheiten zwischen Frauen und Männern und 
konzentriert sich dabei vor allem auf die Asymmetrie im Bereich der Wissenschaft.

Der erste Teil des Beitrags behandelt zunächst das Aufkommen der modernen Prinzipien der Staatsbürgerschaft und die politische Inkorporation von Frauen. Betont werden dabei zum einen die Unterschiede zwischen liberalen und radikalen Auffassungen über den Erwerb der staatsbürgerlichen Rechte für Frauen; zum anderen wird der weltumspannende Charakter dieses Wandels und dessen Beschleunigung in der Zeit nach dem Zweiten Weltkrieg unterstrichen. Die Rechte von Frauen und deren Verletzung sind inzwischen fester Bestandteil eines globalen Themenkatalogs und national-staatlicher Programme. Globale Fortschritts- und Gerechtigkeitsstandards werden oftmals aufgegriffen, um auf den Status von Frauen in der gesamten Welt hinzuweisen.

Im zweiten Teil gehe ich auf die weltweite Institutionalisierung der Wissenschaft ein. Über die Wissenschaft als elitäre Domäne und als Bastion männlicher Privilegien ist bereits viel geschrieben worden. Der institutionelle Charakter der Wissenschaft hat historisch und räumlich unterschiedliche Formen angenommen, zudem variieren der Prestigegrad und die Ebene der männlichen Vorherrschaft. Das wachsende Interesse an geschlechtsspezifischen Fragen im Bereich der Wissenschaft reflektiert den weltweiten Triumph der Wissenschaft als den Entwicklungspfad mit der höchsten Legitimation. In beiden Teilen verweise ich auf international vergleichende Studien, die bezüglich der Staatsbürgerschaft von Frauen, der Entwicklung der Wissenschaft und der Wissenschaft als Entwicklungsmodell internationale Trends aufzeigen.

Der dritte Teil beschäftigt sich mit der Unterscheidung zwischen Frauen in der Wissenschaft und Frauen und Wissenschaft. In der Literatur zu Frauen in der Wissenschaft dominieren Fragen des Zugangs und der Partizipation; sie sind zumeist durch ein liberales Verständnis von Staatsbürgerschaft und ein Verständnis von Gleichheit als Ähnlichkeit (sameness) bestimmt, Dagegen geht die Literatur zu Frauen und Wissenschaft eher von radikalen Perspektiven aus und unterstreicht den Gesichtspunkt, die Wissenschaft zu entmännlichen. Selbstverständlich spielen in diesen Studien Zugang und Partizipation ebenfalls eine wichtige Rolle, aber akzentuiert wird vor allem die Konstitution der Wissenschaft und ihr vermeintlich maskuliner Charakter. Für die Geschlechtersoziologie geht es in der Wissenschaftsforschung vor allem um verbesserte Zugangsmöglichkeiten, Beschäftigung und anerkannte Partizipation und die Umgestaltung der Wissenschaft. 
Frauen in der Wissenschaft - Frauen und Wissenschaft | 28I

\section{Staatsbürgerschaft und die politische Inkorporation von Frauen}

Das Werk von T. H. Marshall kann weiterhin als Ausgangspunkt einer Analyse der Staatsbürgerschaft herangezogen werden, trotz seiner Begrenzungen und der wachsenden Kritik an seiner Konzeptualisierung. Aus seiner Perspektive beinhalten die modernen Prinzipien der Staatsbürgerschaft die Entwicklung ziviler, politischer und sozialer Rechte des Individuums. Historisch waren diese zunächst und in erster Linie gesetzlich verankerte Rechte und Rechtsgleichheit vor dem Gesetz, nicht unbedingt materielle Gleichheit oder Gleichheit der Lebensumstände. Beispiele für zivile und politische Rechte sind das Recht auf freie Meinungsäußerung und auf Versammlungsfreiheit, das Recht, Repräsentanten zu wählen oder sich selbst zur Wahl zu stellen. Diese Rechte werden oft als Abwehrrechte, als Begrenzung der Staatsmacht bezeichnet, da sie es dem Staat verbieten, Maßnahmen zu ergreifen, die die Ausübung dieser Rechte einschränken. Demgegenüber erfordern soziale Rechte Maßnahmen des Staates, die den einzelnen Bürgern ein gewisses Maß an ökonomischer Fürsorge und Sicherheit gewährleisten und ihnen einen bestimmten Anteil am nationalen Reichtum sichern. Diese Rechte werden zumeist unter dem Gesichtspunkt diskutiert, daß sie den Marktmechanismus und private Interessen begrenzen. Beispiele dafür finden sich in den Bestimmungen zur Sozialversicherung, aber auch im Anrecht auf Schulbildung (vgl. Marshall I964; Bendix I964). Die Abgrenzung zwischen den unterschiedlichen Typen von Rechten ist nicht immer eindeutig: Einige Liberale des I9. Jahrhunderts, wie z. B. John Stuart Mill, befürworteten zwar das Recht auf allgemeine Schulbildung, lehnten es aber ab, dafür den Staat in die Pflicht zu nehmen. Heute unterstützen einige Zeitgenossen das Recht der Frauen auf den Zugang zu wissenschaftlichen Ausbildungsplätzen; den Forderungen nach staatlicher Intervention, die die Durchsetzung dieses Rechts erleichtern könnten, stehen sie aber durchaus skeptisch gegenüber. Ob Bildungsrechte zu den sozialen Rechten gehören, ist also zum Großteil davon abhängig, ob der Staat diese Rechte und ihre Umsetzung unterstützt.

Die politische Theorie des I9. Jahrhunderts postulierte das abstrakte Individuum als Träger von bürgerlichen Rechten, das seine öffentlichen Pflichten verantwortungsbewußt erfüllt, obwohl die Staatsbürgerrechte in der Praxis zunächst auf besitzhabende Männer beschränkt blieben. Die Ausdehnung dieser Rechte auf die Arbeiterklasse bzw. auf die unteren Schichten wurde damit begründet, daß auch diese Gruppen als verantwortungsvolle Bürger politisch integriert werden könnten. Die Konstruktion des Nationalstaats als imagined community beinhaltete die Idee einer horizontalen Kameradschaft (horizontal comradeship), also das Konzept eines ka- 
meradschaftlichen Verbunds von Gleichen, der der Staatsbürgerschaft den Weg ebnete (vgl. Anderson 199I). Wenn auch einige Rechte in manchen Ländern früher und vollständiger ausgebildet waren - in Deutschland fand sich frühzeitig eine Betonung der Sozialversicherung, während in den Vereinigten Staaten die zivilen und politischen Rechte zuerst entwickelt wurden -, so kann man doch Komplexe von Staatsbürgerrechten ausmachen, die zunächst in Westeuropa und in großen Teilen des amerikanischen Kontinents institutionalisiert wurden. Heute ähneln sich nationalstaatliche Verfassungen stark in ihrer Betonung von Staatsbürgerrechten, trotz der Verschiedenheit des historischen Erbes, des aktuellen Grades der Industrialisierung, der Urbanisierung und sogar der Formen von politischer Herrschaft (vgl. Boli I987). Zudem werden viele dieser Rechte heute als Menschenrechte deklariert, was für den Staat beides, nämlich weitere Beschränkungen und auch weitere Verpflichtungen mit sich bringen kann. So ist z.B. das Recht auf eine Basisschulausbildung inzwischen Teil der Allgemeinen Erklärung der Menschenrechte. Auf Weltkonferenzen wird dieser Sachverhalt gern ins Feld geführt, um Nationalstaaten zur Sicherstellung einer allgemeinen Schulbildung zu bewegen (vgl. Chabbott 2002).

Die Entstehung moderner Prinzipien der Staatsbürgerschaft ist freilich kein geschlechtsneutraler Vorgang gewesen. Das abstrakte Individuum war männlich, und die vorgestellte Gemeinschaft zog eine scharfe Grenze zwischen dem Privaten und Öffentlichen, eine Grenzziehung, die ihre Lebensfähigkeit in unterschiedlichem Ausmaß auch der Konstruktion von Mutterschaft und Hausfrauendasein verdankt.

Die feministische Kritik an älteren Theorien der Staatsbürgerschaft konstatierte häufig deren Blindheit in bezug auf den Geschlechteraspekt von Bürgerschaft. Für einige Kritiker und Kritikerinnen geht es vor allem darum, daß Frauen nicht dieselben Rechte wie die Männer erhielten. Es ist somit die Ausschlußdimension dieser Rechte, die kritisiert wurde. Die Lösung des Problems bestand dann darin, Frauen ebenso wie Männer als erwachsene Bürger anzuerkennen und sie als eigenständige Personen und aktive Bürgerinnen vor dem Gesetz zu achten. Diese Kritik und die damit verbundene Problemlösung bilden den Kern der liberalen Perspektive, welche bis heute nationale und internationale politische Diskurse und Analy. sen prägt.

Anderen Kritikern und Kritikerinnen geht es jedoch vor allem darum, daß die Menschenrechte faktisch Rechte von Männern, einer Hälfte der Menschheit, waren. Demzufolge sind diese Rechte und die daraus abgeleiteten politischen Maßnahmen begrenzt und entsprechen den Interessen von Männern mehr als denen von Frauen (vgl. Orloff 1993; Pateman 1995). Diese Sicht erfordert einen komplexeren Lösungsansatz, der die Grundlagen der Staatsbürgerrechte, deren Annahmen über die Natur des erwachse- 
nen Individuums und seiner Bedürfnisse hinterfragt. Diesen Ansatz bezeichne ich aufgrund seiner Kritik am modernen Konzept der Staatsbürgerschaft als die radikale Perspektive. Wachsenden Zuspruch findet diese Sicht auch deswegen, weil ihre Vertreter und Vertreterinnen beide Aspekte aufgreifen, d.h., sie wollen die Erfolge des liberalen Konzepts sichern, aber auch über die Ableitung der Staatsbürgerschaft für Frauen aus männlichen Rechten hinausgehen (vgl. O'Connor et al. 1999).

Wie lassen sich nun angesichts der weiten Verbreitung des Topos der fortdauernden Ungleichheit Erfolge im Bereich der Staatsbürgerrechte überhaupt messen? Bislang wurden Zugewinne hier nur behauptet, nicht aber dokumentiert. In diesem Beitrag bieten verschiedene Studien, die transnationale Trends untersuchen, die Grundlage für den Beleg der Zugewinne. Keine dieser Untersuchungen weist nach, daß die Gleichheit der Geschlechter erreicht worden ist. Sie kommen dagegen nur zu dem Ergebnis, daß im Vergleich zu früher die Ungleichheit zwischen den Geschlechtern abgenommen hat. Zudem gelten diese Ergebnisse nur für bestimmte Dimensionen der Ungleichheit. Dies bringt uns wieder zu den Unterschieden zwischen liberalen und radikalen Perspektiven zurück.

Betrachten wir zunächst den Fall des Wahlrechts. Anfang des 20. Jahrhunderts hatten Frauen nur in Neuseeland dieses Recht. Trotz jahrzehntelanger Kämpfe besaßen Frauen weder in Großbritannien noch in den Vereinigten Staaten das Wahlrecht. Im späten Ig. Jahrhundert schienen die Gegner übermächtig, doch am Ende des 20. Jahrhunderts hatten Frauen in 96 Prozent aller Nationalstaaten das Recht zu wählen und gewählt zu werden. Während dieses Zeitraums schrumpfte die zeitliche Distanz, in der zunächst Männern und dann Frauen das Wahlrecht verliehen wurde. So erwarben Frauen und Männer in der zweiten Hälfte des 20. Jahrhunderts das Wahlrecht fast immer gleichzeitig. Das frühere Muster, nach dem Frauen das Wahlrecht später als Männer erkämpften, setzte sich nicht mehr einfach fort. In den neuen Nationalstaaten, die nach dem Zweiten Weltkrieg entstanden, bildete die gleichzeitige Verleihung des Wahlrechts das vorherrschende Muster. Dort, wo es von repressiven Regimes wieder entzogen wurde, geschah dies für beide Geschlechter, nicht nur für Frauen. Aus dem Wahlrecht, das einst umstritten gewesen war, wurde schließlich eine Selbstverständlichkeit des politischen Lebens.

Zudem änderten sich historisch die Faktoren, die die Verleihung des Wahlrechts beeinflußten. In der ersten Hälfte des 20 . Jahrhunderts waren es interne soziale Unterschiede der Nationalstaaten, die den Zeitpunkt der Einführung bestimmten, später wurden internationale Verbindungen und Einflüsse wichtiger. So hatte zwischen 1890 und I940 der Grad der Verwestlichung eines Landes einen positiven Effekt auf die Verleihung des Frauenwahlrechts, danach aber spielten regionale Politik und weltweite Einflüsse 
eine größere Rolle (vgl. Ramirez et al. 1997). Nationale Geschlechterpolitik orientierte sich nun zunehmend an internationalen Standards und weniger an lokalen Bedingungen oder an dem spezifischen historischen Erbe.

Die weltweite Anerkennung dieses politischen Rechts für Frauen kann nicht hoch genug bewertet werden. Es ist Teil einer weltweiten Transformation, in der internationale Organisationen zunehmend die Rechte von Frauen auf ihre Agenda setzen. So untersucht z. B. Berkovitch (1999) die veränderte Haltung der Internationalen Arbeitsorganisation (IAO) in Debatten über den Status von Frauen. Die frühere, protektionistische Gesetzgebung wurde abgelöst durch ein Verständnis von Frauen als erwachsene Bürgerinnen, die Anspruch auf Gleichheit bei der Einstellung und auf gleichen Lohn für gleiche Arbeit haben. Die nationale Gesetzgebung verlief oft parallel zu internationalen Erklärungen, und auf nationaler und internationaler Ebene wurden geschlechtsspezifische Argumentationen zur Rechtfertigung der Ungleichbehandlung von Frauen am Arbeitsplatz entkräftet. Berkovitch (1999) sieht in diesem Wandel eine entscheidende Akzentverschiebung eines Verständnisses von Frauen als Mütter, die auf besonderen Schutz angewiesen sind, hin zu Frauen als Individuen, die gleiche Chancen beanspruchen. In den letzten Jahrzehnten geht dieser Wandel manchmal einher mit Auffassungen von Frauen als Humankapital und als unerschlossene nationale Ressource, die für die Entwicklung des Landes unverzichtbar ist. Doch diese Auffassungen beinhalten auch ein Verständnis von Gerechtigkeit und fairer Behandlung. Hierbei wird oft stillschweigend angenommen, daß Gerechtigkeit und faire Behandlung darin bestehen, Frauen genauso wie Männer zu behandeln.

Parallele Entwicklungen sind im Bildungssystem zu beobachten. Die früheren Debatten über den Sinn von Bildung für Frauen sind inzwischen obsolet geworden. Die absoluten Zahlen von Mädchen und Frauen im Schul- und Bildungswesen haben auf allen Ebenen zugenommen. Außerdem lassen sich Zuwachsraten in den entsprechenden Altersgruppen der Bildungsstufen feststellen sowie ein relativer Anstieg von Schülerinnen und Studentinnen im Vergleich zu der Anzahl männlicher Schüler und Studenten. Besonders auffallig ist in den letzten Jahrzehnten die weltweit wachsende Präsenz von Frauen im höheren Bildungswesen; ein Großteil dieses Anstiegs kann nicht als Resultat endogener sozialer Faktoren erklärt werden (vgl. Bradley/Ramirez 1996). Die Debatten über Geschlecht und Bildung haben sich ebenfalls verschoben. Sie weisen ein ähnliches Muster auf, wie es Berkovitch im Bereich der ökonomischen Rechte untersucht hat. Früher vertraten die Verfechter einer Zulassung von Mädchen und Frauen an Schulen und Universitäten ihre Position mit dem Argument, daß Bildung aus ihnen gute Mütter machen würde, die ihre Kinder richtig erziehen würden. Heute ist die Argumentation viel stärker auf Frauen als Individuen 
Frauen in der Wissenschaft - Frauen und Wissenschaft | 285

bezogen. Auch nach Geschlecht differenzierte Lehrpläne waren um die Jahrhundertwende die Norm, während sich die Curricula heutzutage offensichtlich mehr an Gleichheit orientieren (vgl. Ramirez/Cha 1990). Selbst die einführenden Textbücher für naturwissenschaftliche Schulfächer beziehen Mädchen inzwischen stärker ein (vgl. McEneaney/Meyer 1999). Es ist nicht überraschend, daß diese individualistischere Argumentation sich zudem auch auf Begriffe wie Humankapital und Menschenrechte stützt. Der persönliche Entwicklungsprozeß ist zu einem Menschenrecht geworden, und es wird allgemein angenommen, daß diese Entwicklung im größeren Rahmen auch zum nationalen Fortschritt beiträgt.

Diese Veränderungen im politischen Gemeinwesen, in der Wirtschaft und im Bildungssystem, dies muß betont werden, beziehen sich auf den Grad des Zugangs und der Partizipation. Ebenso wichtig ist der Aspekt, daß diese Rechte Männern bereits früher eingeräumt wurden. Der Erfolg einer ganzen Reihe von Frauenbewegungen bestand darin, die Anerkennung von Frauen als Bürgerinnen mit Bürgerrechten zu erreichen. Die politische Inkorporation von Frauen erfolgte dabei unter der Kategorie des abstrakten Individuums. Heute werden diese Erfolge oft übersehen oder trivialisiert, da angesichts des inzwischen vorherrschenden Gleichheitsstandards selbst Vertreter einer liberalen Perspektive höhere Erwartungen entwickeln. Denn heute geht es nicht mehr nur darum, ob Frauen wählen dürfen, sondern wieviele Frauen in Machtpositionen gelangen. Es geht nicht nur um gleiche Chancen für Frauen auf dem Arbeitsmarkt, sondern um ihren beruflichen Statusgewinn. Nicht nur die wachsenden Zahlen von Frauen im höheren Bildungswesen sind von Interesse, sondern ihre Positionen in prestigereichen Disziplinen und an angesehenen Universitäten. Dieses historische Muster des Zugewinns und der inzwischen etablierte Gleichheitsstandard bilden den Maßstab für höhere Erwartungen und führen dazu, daß bestimmte Mängel nicht nur als Ungleichheit, sondern auch als Ungerechtigkeit wahrgenommen werden. In sozialpsychologischen experimentellen Studien über Gerechtigkeit ist das Prinzip, das dieser Wahrnehmung zugrunde liegt, gut belegt worden: Die Wahrnehmung von Ungerechtigkeit resultiert nicht einfach aus Ungleichheiten, sondern wird gerade dann wahrscheinlich, wenn Gleichheitserwartungen enttäuscht werden. Derselbe Grad der Ungleichheit wurde - abhängig vom Umfang egalitärer Erwartungen - unterschiedlich wahrgenommen und bewertet.

Aus radikaler Perspektive geht es nicht um die Frage, ob der Kreis von Personen mit bestimmten Rechten bereits um Frauen erweitert wurde. Im Mittelpunkt steht vielmehr der Inhalt dieser Rechte selbst. Die radikale Perspektive rückt Frauen in ihrer spezifischen Körperlichkeit in den Mittelpunkt. Sie legt den Akzent auf Frauen als verkörperte Individuen (bodied individuals) und betont das weibliche Recht auf körperliche Integrität und 
Selbstbestimmung. Hervorgehoben werden dabei z. B. die reproduktiven Rechte und der Kampf gegen Mißbrauch und Gewalt gegen Frauen, und zwar auch innerhalb der Familie. Thema sind also nicht abgeleitete Rechte, also nicht Rechte, über die Männer bereits früher verfügten. Es handelt sich vielmehr um Frauenrechte, die Frauen als Frauen beanspruchen. Noch wichtiger ist vielleicht, daß es aus dieser Perspektive die Spielregeln selbst sind, die in Frage gestellt werden. Inklusion bleibt zwar weiterhin ein wichtiges Thema, doch nun wird das Konzept der Inklusion zur Debatte gestellt. Radikale Ansätze gehen davon aus, daß das abstrakte Individuum faktisch ein männliches ist. Von Frauen wurde demzufolge im Zuge ihrer politischen Inkorporation implizit verlangt, wie Männer zu sein, um von Staatsbürgerschaft und sozialpolitischen Regelungen in gleichem Maße zu profitieren (vgl. Orloff I993; Okin I989). Aus dieser Perspektive ist somit eine grundlegende Revision der Logik der Staatsbürgerschaft notwendig.

Für jene Rechte, die Männern zuerst gewährt wurden, lassen sich, und das ist nicht verwunderlich, mehr Belege in international vergleichenden Studien finden. Der Inkorporationsgrad von Frauen im Bildungswesen, in der Politik und auf dem Arbeitsmarkt hat allgemein zugenommen, und der historische Widerstand dagegen befindet sich oftmals im Konflikt mit dem Recht und sogar mit der öffentlichen Meinung. Der Grad der Einbeziehung variiert von einem Land zum anderen, doch es ist auffällig, wieviel mehr Frauen sich im Bereich der Politik engagieren und dort auch beschäftigt sind. Bei der Diskussion von Frauen in der Wissenschaft wird deutlicher werden, daß die gegenwärtigen Gleichheitserwartungen weit über die bloße Zulassung, Beschäftigung und Tätigkeit hinausgehen.

Wenden wir uns für einen Moment dem Status jener Rechte zu, die Frauen als Frauen beanspruchen. Reproduktive Rechte sind hierfür ein naheliegendes Beispiel. Sind diese Rechte tatsächlich zugestanden worden und haben sie denselben selbstverständlichen Status erlangt wie die abgeleiteten staatsbürgerlichen Rechte, also das Wahlrecht? Diese Frage wird in einem Aufsatz ausführlicher behandelt, der die Liberalisierung der Abtreibung in verschiedenen Ländern mit den Trends bei der Vergabe des Wahlrechts vergleicht (vgl. Ramirez/McEneaney 1997). Zwei Ergebnisse sind hier besonders erwähnenswert. Zuerst geht der weltweite Trend dahin, Gesetze zur Abtreibung weiter zu liberalisieren. Dies mag angesichts der andauernden Abtreibungskontroversen in den Vereinigten Staaten erstaunlich scheinen. Es wäre jedoch falsch anzunehmen, daß weltweite Trends in direktem Zusammenhang mit Mustern in den Vereinigten Staaten stehen und von diesen beeinflußt werden. Geht man statt dessen davon aus, daß globale Einflüsse auf verschiedenen Ebenen wirksam sind, u.a. durch eine Reihe relativ autonomer nicht-staatlicher Organisationen, so ist die Ausbreitung dieses besonders auf Frauen bezogenen Rechts nicht sonderlich über- 
Frauen in der Wissenschaft - Frauen und Wissenschaft | 287

raschend. Der zweite Befund ist jedoch, daß dieses Recht rückläufig ist. Rumänien liefert das extremste Beispiel dafür, wie Veränderungen des politischen Regimes zu Änderungen in der Abtreibungsgesetzgebung führen können. Eine staatlich beschlossene Begrenzung des Rechts auf Selbstbestimmung stellt die Ein-Kind-Regelung in China ebenfalls dar, wenn auch hier die Abtreibung verordnet statt verboten wird. Abtreibungsrechte sind heutzutage kontroverser und gefährdeter als Wahlrechte oder andere abgeleitete Staatsbürgerrechte. Dennoch spricht der weltweite Trend für eine langsame Bestätigung auch dieser Rechte, die nicht auf der männlichen Staatsbürgerschaft beruhen.

Mit den reproduktiven Rechten ist eine Reihe von staatsbürgerlichen Forderungen verknüpft, die - von der sexuellen Belästigung bis hin zur Vergewaltigung - Mißbrauch und Gewalt gegen Frauen thematisieren. Es liegt kaum Literatur vor, die diese Fragen im Ländervergleich behandelt, um Trends in der Gesetzgebung oder der öffentlichen Meinung zu identifizieren. Doch scheinen diese Themen auf globaler Ebene und bei internationalen Konferenzen zunehmend eine wichtigere Rolle spielen. Die Phasen dieses Wandels erstrecken sich von der geschlechtsneutralen Erklärung der Menschenrechte der Vereinten Nationen im Jahr 1948 über die abgeleitete Konvention über die Politischen Rechte der Frau im Jahr 1952 über die 1979 offener formulierte Konvention über die Beseitigung jeder Form von Diskriminierung der Frau bis hin zur sehr spezifischen Konvention über die Beseitigung von Gewalt gegen Frauen im Jahr 1994. Für die Bekämpfung der sexuellen Belästigung von Frauen zieht man den Menschenrechtsdiskurs heran, und dies sogar in Kontexten, in denen Frauen historisch kaum individuelle Rechte eingeräumt wurden wie in Japan (vgl. Tiberghien 2000). Selbstverständlich wäre es naiv zu behaupten, daß diese Erklärungen der Vereinten Nationen zu einer tatsächlichen Reduzierung des Mißbrauchs und der Gewalt gegen Frauen führen. Die Konventionen sind vielmehr als ein globaler kultureller Rahmen zu verstehen, in dem Praktiken männlicher Dominanz als verletzende und gewalttätige Verstöße gegen die Menschenrechte von Frauen klassifiziert werden können. Folglich tragen die Konventionen dazu bei, Bedingungen politischer Inklusion zu problematisieren.

Auf nationaler Ebene bestehen ähnliche Entwicklungen, z.B. in der Einrichtung von Ministerien oder administrativer Stellen, die sich mit Frauenfragen beschäftigen. Am Ende des Zweiten Weltkriegs verfügten nur io Prozent der damals existierenden Staaten über solche Stellen. Bis 1970 hatte sich diese Zahl verdreifacht und schnellte bis I980 sogar auf 80 Prozent (vgl. Berkovitch 1999). Hier ist wiederum nicht entscheidend, daß sich die realen Zustände auf allen Ebenen tatsächlich verbessert haben, sondern daß Themen politisch relevant werden, sobald öffentliche Ämter sich damit befassen. Außerdem sind es nicht nur nationale Behörden, die sich als Orga- 
nisationen dieser Themen annehmen. Auf globaler Ebene hat die Anzahl von internationalen Nicht-Regierungsorganisationen zugenommen, die sich mit Frauenfragen befassen, und ihre Anstiegsrate ist höher als die anderer internationaler Nicht-Regierungsorganisationen (vgl. Boli/Thomas 1997). Die Anzahl der I3 Nicht-Regierungsorganisationen, die im Jahr I975 Frauenrechte vertraten, explodierte auf 265 im Jahr 1995 (vgl. Tiberghien 1997).

Abbildung 1: Nicht-Regierungsorganisationen 1895-1995

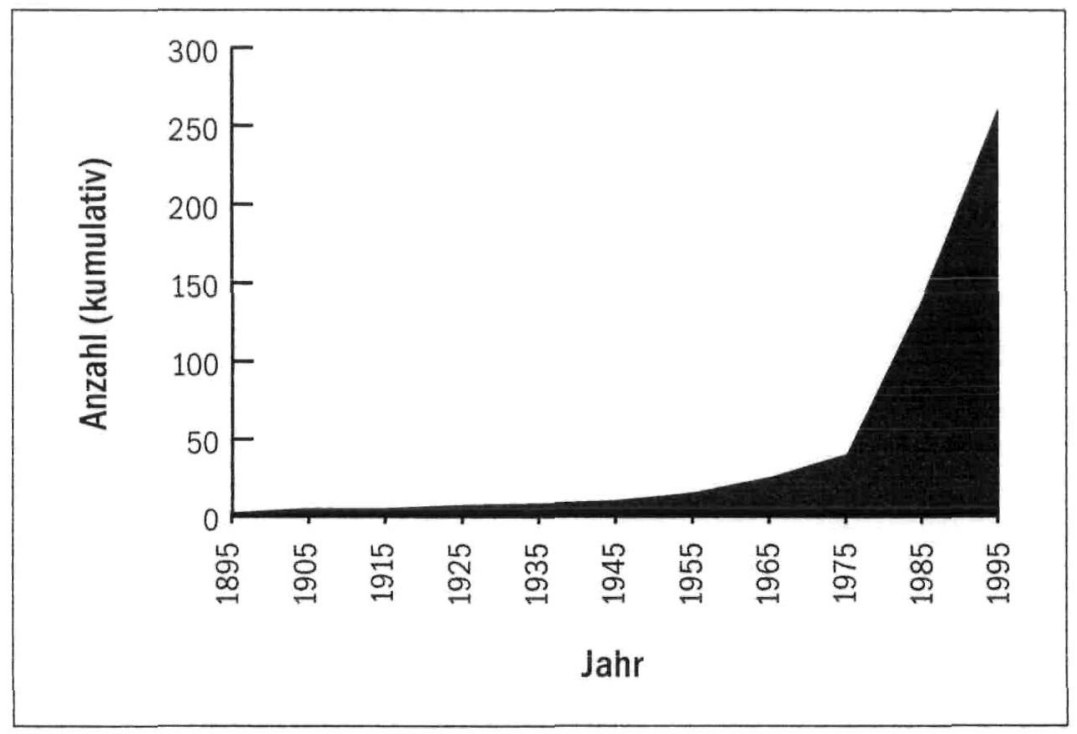

Was zeigt nun die international vergleichende Forschung insgesamt? Zunächst kommt sie zu dem Ergebnis, daß während des gesamten 20. Jahrhunderts weltweit Trends zur Ausweitung von staatsbürgerlichen Rechten für Frauen bestehen. Zweitens dokumentiert diese Forschung, daß die Zugewinne deutlicher und unumstrittener waren, wenn die betreffenden Rechte aus männlichen Rechten abgeleitet waren. Drittens erweist sich, daß auf globaler Ebene und auf internationalen Konferenzen Frauenrechte inzwischen als Rechte von Frauen stärker betont werden. Doch diese Rechte sind kontroverser und laufen eher Gefahr, wieder entzogen zu werden. Schließlich zeigt sich auch in den Debatten nationaler und internationaler Entwicklungshilfe-Organisationen ein gesteigertes Interesse an Frauenrechten und themen. In diesem anwachsenden Diskursfeld werden Gleichheitsstandards entwickelt und ausgedehnt, mit deren Hilfe wiederkehrende, aber auch neue Formen geschlechtlicher Ungleichheit leichter identifiziert werden können. Weltweit verbreitete Fortschritts- und Gerechtigkeitsmodel- 
le prägen diesen Diskurs, der Nationalstaaten, internationale Organisationen und auch soziale Bewegungen gleichermaßen beeinflußt.

Der große Teil dieser Debatten ist bereits seit geraumer Zeit universalistisch. Frauen beschränken sich bei der Forderung von Rechten nicht auf ihr eigenes Land, sondern sprechen weltweit alle Frauen als Nutznießerinnen von Rechten an. Selbst dann, wenn Feministinnen in den weniger entwickelten Ländern die westlichen Spielarten des Feminismus als eurozentrisch angreifen, berufen sie sich dabei auf universalistische Standards, die in den globalen Fortschritts- und Gerechtigkeitsmodellen verankert sind. Alle Völker, so wird argumentiert, haben ein Anrecht auf ihr historisches Erbe und ihre eigenen Traditionen, und zwar auch auf jene, die eine stärkere geschlechtliche Differenzierung beinhalten als im Westen akzeptiert. Es sind universalistische Ideen von nationaler Souveränität und kultureller Autonomie, die in diese Kritik eingehen, bzw. zumindest in solche, die die Widerlegung relativ gut überstehen und Bestand haben. Kultur kann folglich als akzeptables Argument benutzt werden, um Zweifel über ein koedukatives Bildungswesen zu äußern, nicht aber um gleiche Bildungschancen für Frauen in Frage zu stellen. In Saudi-Arabien wurde auf diese Weise die Trennung der Geschlechter im Bildungswesen beibehalten, während der Anteil von Frauen im höheren Bildungswesen sich gleichzeitig ausgeweitet hat (vgl. Bradley/Ramirez I996). Diese Ausdehnung und weitere Veränderungen des Staatsbürgerstatus von Frauen deuten darauf hin, daß sich darin eine nationale Verpflichtung zu Fortschritts- und Gerechtigkeitsmodellen manifestiert, die alle redlichen und konformen Nationalstaaten anstreben.

Nationale Rechtschaffenheit ist ein wichtiges Thema, das im Rahmen dieses Beitrags nicht erschöpfend behandelt werden kann. Nationalstaaten und solche, die es werden möchten, operieren nicht in einem Vakuum. Sie können ihre Nationalstaatlichkeit nicht nach eigenen Vorstellungen oder der ihrer Eliten improvisieren. Für den Nationalstaat sind vielmehr Skripte verfügbar, die auf unzähligen internationalen Konferenzen und in Organisationen von Professionellen und Experten formuliert werden. Vieles davon hat einen beratenden Charakter, wobei der Rat hinreichend abstrakt ist, um international unterschiedlich interpretiert und umgesetzt zu werden. Doch der zunehmende Isomorphismus der Nationalstaaten, d. h. ihre strukturelle Ähnlichkeit, wäre kaum zu erklären, wenn man für die Formation von Nationalstaaten nicht gemeinsame Modelle und Baupläne postulierte. Besonders augenfällig ist diese Ähnlichkeit bei den formalen Prinzipien, die offizielle staatliche Maßnahmen und Organisationen für ihre Politiken als Rationalitätsimperative benutzen. Aber auch in vielen konkreten Praktiken gleichen sich die Ziele von Nationalstaaten mit der Zeit einander an (vgl. Meyer et al. I997). Die herausragende Bedeutung wissenschaftlicher Autorität, die diesen globalen kulturellen Modellen innewohnt, kann kaum über- 
schätzt werden. Es ist diese Autorität der Wissenschaft, um die es im folgenden geht.

\section{Die Globalisierung der Wissenschaft}

Sowohl Kritiker als auch Befürworter erkennen den weltweiten Siegeszug der wissenschaftlichen Autorität an. Für die Befürworter haben die inhärente Nützlichkeit der Wissenschaft und die wissenschaftliche Forschung dazu geführt, daß andere, nicht-wissenschaftliche Realitätsbezüge weitgehend verdrängt wurden. Wissenschaft ist daher für die Gesellschaft in einem hohen Maße zweckdienlich, so daß ihre Autorität anerkannt und belohnt wird. Autoren, die in dieser Tradition stehen, halten diese Entwicklung für wünschenswert und beklagen jede noch so kleine Schwankung des öffentlichen Vertrauens in die Wissenschaft als Ignoranz.

In der Sicht der Kritiker sind es ökonomisch oder wissenschaftlich dominante Gruppen bzw. Klassen, die die Wissenschaft für ihre eigenen Interessen benutzen. Manchmal liegt die Macht sogar bei den Wissenschaftlern selbst, die erfolgreich Kräfte mobilisieren, um andere soziale Gruppen auszustechen. Die Kritiker stellen die Nützlichkeit der Wissenschaft zwar in Frage, aber sie erklären weder die Legitimität der Wissenschaft noch die Autorität von Wissenschaftlern, eine Autorität, die oftmals die Ressourcen von einzelnen Wissenschaftlern bei weitem übersteigt. Während die Befürworter der Wissenschaft die Nützlichkeit vieler wissenschaftlicher Anwendungen beweisen, bleibt bei ihnen erklärungsbedürftig, warum ein großer Teil der Wissenschaft nicht nutzenorientiert ist. Sie übersehen die kulturelle Funktionsweise der Wissenschaft, nämlich ihre Autorität, die in der kulturellen Konstitution der Wissenschaft als rationaler und rationalisierender Vorgang begründet ist. Diese kulturelle Geltung der Wissenschaft wird eher von ihren Kritikern erkannt, die dann allerdings fälschlichweise annehmen, daß Wissenschaft aufgrund ihrer kulturellen Bedeutung nicht funktionieren könne.

Aus der Perspektive des Weltgesellschaftskonzepts ist der hohe Legitimationsgrad entscheidend, durch den die Wissenschaft charakterisiert ist. Diese Legitimation beruht darauf, daß die Wissenschaft Zugang zu weitverbreiteten Entwicklungsmodellen, nämlich zu Fortschritt und Gerechtigkeit, verschafft (vgl. Drori et al. 2002). Folglich wird erwartet, daß wissenschaftliche und technologische Entwicklungen das wirtschaftliche Wachstum unmittelbar ankurbeln. Wissenschaftliche und technische Prozesse sollen entscheidend zur Entwicklung des sozialen und politischen Fortschritts beitragen. Als rationale Kultur führt Wissenschaft demzufolge zu einer verbesserten Gesundheitspflege, zu stabileren demokratischen Systemen, zu ei- 
nem vernünftigeren Umgang mit der Umwelt und zu verbesserten Organisationsstrategien wie z.B. in der Verwaltungswissenschaft. Drori (I997) zeigt, daß wissenschaftliche Entwicklungen tatsächlich in einem positiven Zusammenhang mit vielen Indikatoren des sozialen und politischen Fortschritts stehen, wie etwa mit Maßnahmen zur Verbesserung des staatsbürgerlichen Status von Frauen. Ihre Ergebnisse legen nahe, daß viele Aspekte der Wissenschaft durch allgemeine und oftmals nicht-utilitaristische Merkmale bestimmt sind.

Sicherlich gibt es naturwissenschaftliche Anwendungen, die für eine Reihe von Umweltkatastrophen und ihre Folgen verantwortlich gemacht werden, z.B. für die Verschmutzung der Luft oder von Flüssen und ihre Auswirkungen auf den Menschen. Doch generell besteht die Erwartung, daß die Versäumnisse der industriellen oder militärischen Wissenschaft durch andere wissenschaftliche Projekte in den Bereichen Umwelt, Gesundheit und Bildung aufgefangen werden können. Die kulturell bevorzugte und politisch effektivste Antwort auf Probleme, die durch verfehlte Anwendungen von Wissenschaft entstanden sind, ist eindeutig der Appell an die sgute Wissenschaft, die sschlechte $<$ zu korrigieren. Andere denkbare Antworten, wie z. B. die grundsätzliche Ablehnung wissenschaftlicher Autorität oder die lokal verankerte, kulturelle oder historische Skepsis gegenüber wissenschaftlichen Expertisen werden dagegen international kaum Gehör finden.

Die Autorität der Wissenschaft ist in der Schule und in der gesamten Gesellschaft breit akzeptiert. Staatliche Regierungsstellen sind daran interessiert, wie Schüler in Mathematik und naturwissenschaftlichen Fächern abschneiden und wie die Leistungen von Mädchen im Vergleich zu denen der Jungen ausfallen. Veränderungen der Lehrpläne und Ausbildungsreformen zielen auf die Verbesserung der naturwissenschaftlichen Ausbildung ab. Staatliche Stellen erheben Daten über die Belegung natur- und technikwissenschaftlicher Fächer, und die Statistik zu Studienabschlüssen in diesen Disziplinen hat große politische Bedeutung. Die Ergebnisse werden mit der Anzahl von Wissenschaftlern und Ingenieuren in der Arbeitswelt verglichen und mit dem zu erwartenden zukünftigen Bedarf an Fachkräften. Wenn Diskrepanzen vorliegen, werden Initiativen gestartet, um begabte Jugendliche in natur- und technikwissenschaftliche Studien- und Unternehmensbereiche zu führen. In den Vereinigten Staaten vergibt die $\mathrm{Na}$ tional Science Foundation besondere Stipendien für Frauen und Minderheiten, die sich für natur- und ingenieurwissenschaftliche Fächer an den Hochschulen interessieren. Schließlich sind viele Indikatoren entwickelt worden, um wissenschaftliche Produktivität in Form eines Publikationsund Zitationsindexes wissenschaftlicher Aufsätze, Erfindungen und Patente zu messen. 
Das staatliche Interesse an Daten und Reformen wird durch die Annahme geleitet, daß zwischen den Schulleistungen in naturwissenschaftlichen Fächern und der wissenschaftlichen Entwicklung in der Gesellschaft kausale Verbindungen bestehen. Die wissenschaftliche und technische Entwicklung soll wiederum das nationale Wirtschaftswachstum ankurbeln. Implizit findet sich dieser Gedanke in vielen Regierungsberichten über Schulen und die nationale Wirtschaftslage. Abbildung 2 verdeutlicht die Rolle, die Wissenschaft und Technologie für die nationale Entwicklung beigemessen wird. Sie hebt die Verbindungen zwischen naturwissenschaftlichem Grundverständnis, wissenschaftlicher Kompetenz auf dem Arbeitsmarkt und nationalem Wirtschaftswachstum hervor.

Abbildung 2: Die Bedeutung von Wissenschaft und Technologie für nationale Entwicklungen

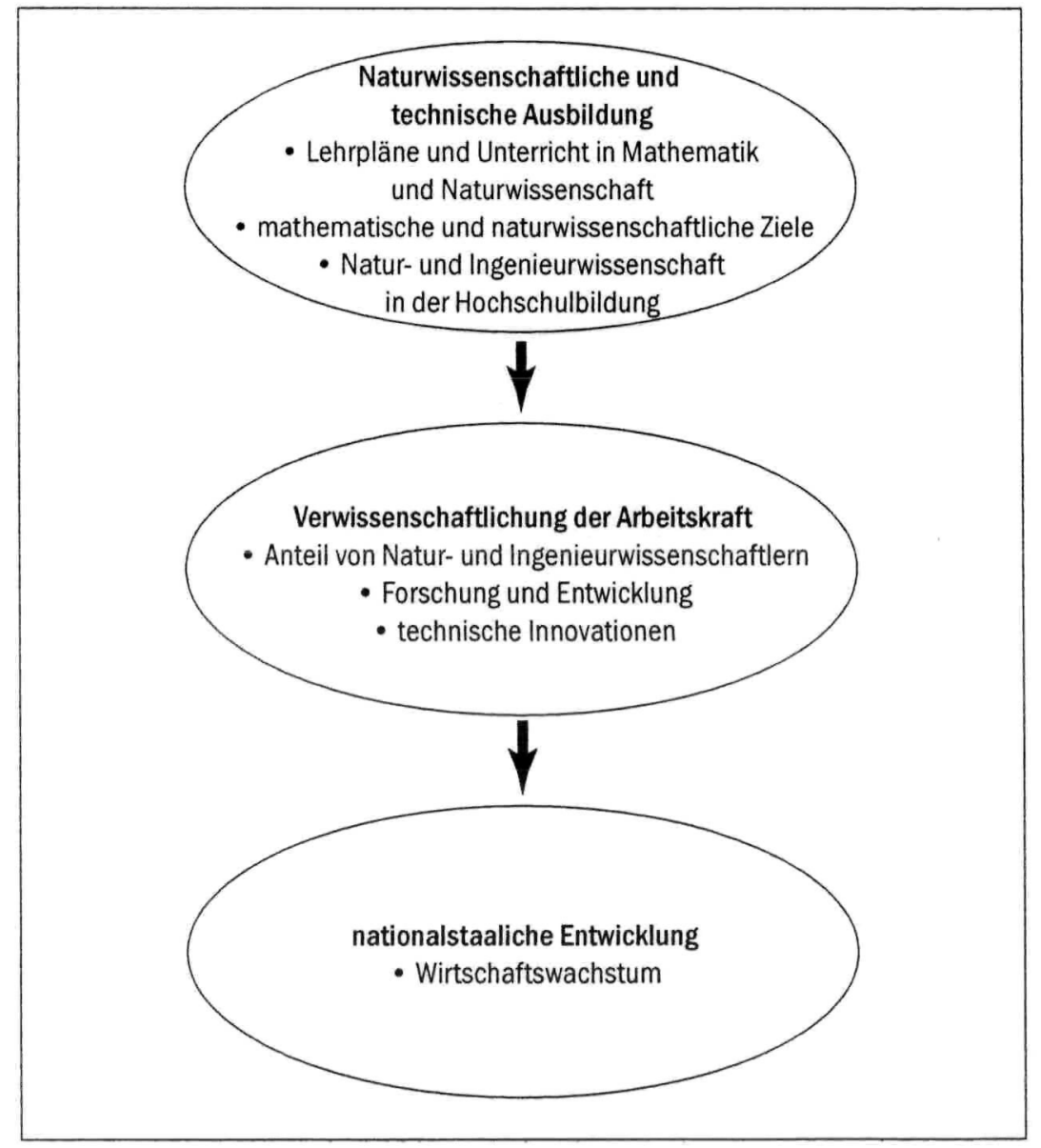


Frauen in der Wissenschaft - Frauen und Wissenschaft | 293

Für diese Zusammenhänge liegen kaum gesicherte Daten vor. Aber das verbreitete Interesse an Wissenschaft als Entwicklungsfaktor hat zur Einrichtung von umfangreichen, international vergleichenden Datenbanken geführt, die verschiedene Indikatoren für die wissenschaftliche Entwicklung berücksichtigen. Eine Langzeitstudie, die die Folgen wissenschaftlicher Entwicklung für das ökonomische Wachstum in verschiedenen Ländern untersucht, hat unterschiedliche Effekte festgestellt. Einige Aspekte der wissenschaftlichen Entwicklung haben tatsächlich positive Auswirkungen auf das Wirtschaftswachstum, wie Human-Kapital-Ansätze und Modernisierungstheorien voraussagen, während andere dagegen negative Konsequenzen haben. Danach wirken sich große Investitionen in kostenintensiven Elitebereichen der wissenschaftlichen Forschung zumindest kurzfristig negativ auf das Wirtschaftswachstum aus, und zwar insbesondere in den Forschungsfeldern, die einen geringeren Bezug zur wirtschaftlichen Produktion haben (vgl. Schofer et al. 2000). Außerdem hat sich die Wissenschaft auf nationaler und internationaler Ebene in weite Bereiche der Forschung und Organisationen ausgebreitet. Das Vertrauen in die Wissenschaft als Entwicklungsmodell übersteigt diesen Studien zufolge bei weitem die empirische Grundlage für solche Zuversicht. Dieses Vertrauen bezieht sich nicht nur auf das wirtschaftliche Wachstum, sondern auch auf den weiteren Bereich sozialer und politischer Probleme.

Historisch hat das Vertrauen in die Wissenschaften zugenommen, auch wenn man die weltweite Ausbreitung wissenschaftlicher Aktivitäten mit berücksichtigt. Abbildung 3 identifiziert einige Indikatoren für wissenschaftliche Aktivität und stellt ihre Ausdehnung im 20. Jahrhundert dar. Auffallend ist der Zuwachs an Ländern, die über nationale Wissenschaftsministerien und/oder staatliche wissenschaftspolitische Einrichtungen verfügen. Zudem läßt sich ein Anstieg zwischen-staatlicher Wissenschaftsorganisationen, internationaler Wissenschaftsverbände sowie ein Anstieg der Mitgliedschaft von Nationalstaaten im International Council of Scientific Unions feststellen. Schließlich zeigt Abbildung 3 auch einen relativ neuen, bescheidenen Zuwachs an Organisationen, die sich der wissenschaftlichen Ausbildung widmen. Diese Trends weisen darauf hin, daß die Welt als Ganzes zunehmend durch nationale und internationale wissenschaftliche Organisationen strukturiert wird. Wissenschaft ist zum Aktionsfeld staatlich-nationaler und internationaler Bürokratien geworden; zugleich bildet sie auch einen wichtigen Bezugspunkt für Verbandsgründungen. Diese Zusammenschlüsse steuern zunehmend weitgefaßte Entwicklungsziele an, liefern wissenschaftliche Positionen zu immer mehr Themen und führen Experten zusammen, deren Rat überall auf der Welt gefragt ist.

Ein Teil dieser Expertenvorschläge spezialisiert sich auf den Status von 
Abbildung 3: Indikatoren für wissenschaftliche Aktivitäten im 20. Jahrhundert

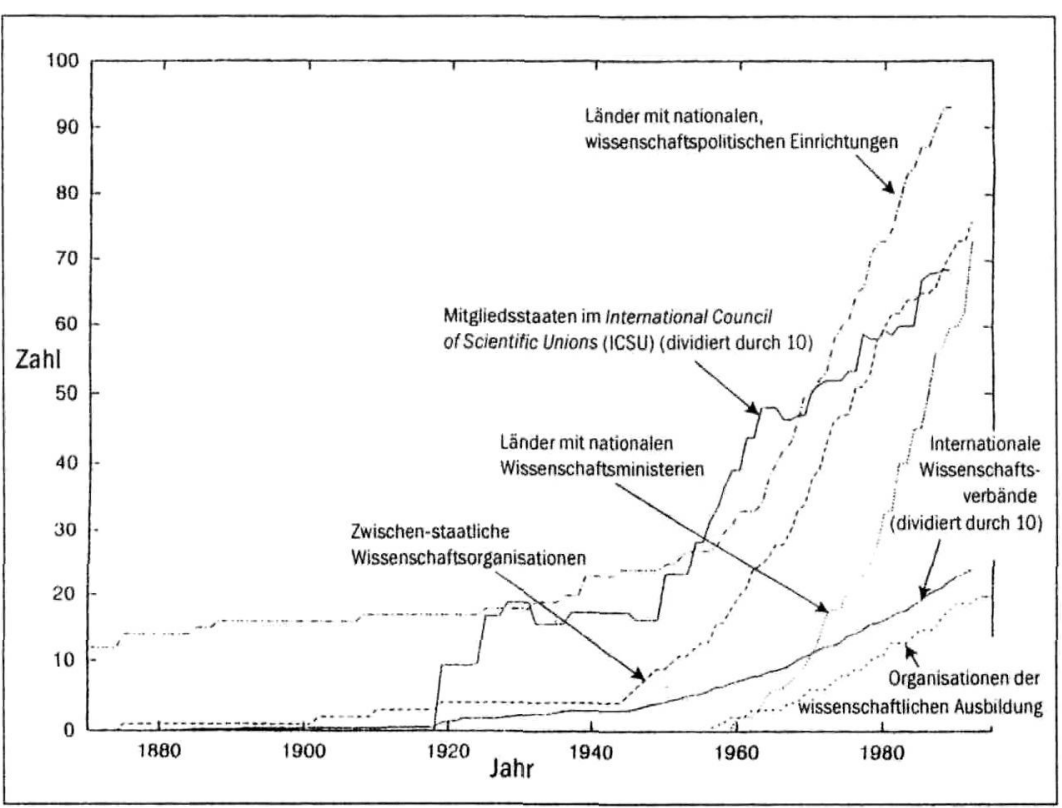

Frauen und auf Frauenfragen. In öffentlichen Diskussionen über die Dimensionen des Status von Frauen werden ebenso wie in politischen Diskursen zahlreiche Indikatoren herangezogen, die sich auf eine Vielzahl von international vergleichenden Daten zur Wissenschaft stützen. Diese Omnipräsenz wissenschaftlicher Autorität, ihre Mystik als elaborierte Form der Rationalität und die verbreitete Tendenz, formale Rationalität mit Männlichkeit gleichzusetzen, haben dazu geführt, daß die feministische Kritik sich in letzter Zeit zunehmend mit der Wissenschaft und bestimmten Wissenschaftsbereichen beschäftigt. Zum Großteil erfolgen diese Untersuchungen aus einer liberalen Perspektive. Sie diskutieren zumeist Fragen der relativen Unterrepräsentanz von Frauen in der Wissenschaft. Im Gegensatz dazu kritisieren radikalere Ansätze Wissenschaft als männliche Institution und befassen sich mit dem Verhältnis von Frauen zur Wissenschaft.

\section{Frauen in der Wissenschaft - Frauen und Wissenschaft}

Im folgenden geht es zunächst um verschiedene Fragen, die aus der liberalen Perspektive von Frauen in der Wissenschaft angesprochen werden und um die entsprechenden Studien. Anschließend werden Fragen behandelt, die der eher radikale Frauen-und-Wissenschaft-Ansatz diskutiert. Beide An- 
sätze sollen nicht beurteilt, sondern ihre Differenzen und folglich auch ihre eventuellen Übereinstimmungen dargelegt werden.

Aus liberaler Perspektive geht es unter anderem um folgende Themen: I. die Erfolge von Mädchen in Mathematik und Naturwissenschaften im Vergleich zu denen von Jungen; 2. die Zugangsmöglichkeiten von Mädchen zu fortgeschrittenen Bildungsangeboten in diesen Fächern; 3. der Anteil von Frauen an der Studentenschaft in natur- und ingenieurwissenschaftlichen Studiengängen; 4. der Anteil von Frauen in den natur- und ingenieurwissenschaftlichen Berufen. Dabei wird oftmals folgender Kausalzusam. menhang hergestellt: Angesichts ihrer Unterrepräsentanz in den entsprechenden Studiengängen sind Frauen auch in diesen Berufen unterrepräsentiert. Die Untervertretung hängt wiederum mit den unterschiedlichen schulischen Ausbildungsmustern zusammen, die den weiblichen Leistungsrückstand entweder reflektieren oder ihn aber überhaupt erst begründen. Damit gibt es auf der beruflichen oder auf der Bildungsebene ein Nachschubproblem (pipeline problem), das für die niedrigeren Zahlen von Studentínnen und/oder weiblichen Berufstátigen verantwortlich ist (vgl. Hanson et al. 1996). Dieses Problem wird oftmals unter dem Aspekt der Nichtausschöpfung des weiblichen Humankapitals oder der mangelnden Chance auf soziale Fairneß und Gerechtigkeit behandelt. Zwei weltweite Tendenzen tragen dazu bei, die positionalen Diskrepanzen zwischen Frauen und Männern als ernsthaftes Problem wahrzunehmen und zu definieren: Die Globalisierung der Wissenschaft als Entwicklungsmodell (science for development) und die Universalisierung egalitärer Geschlechterstandards. Das Vertrauen in die Wissenschaft als Entwicklungsmodell führt zu der Effizienzüberlegung, auch das weibliche Potential in den für die Entwicklung wichtigen wissenschaftlichen Berufen zu nutzen. Der Siegeszug von Gleichberechtigungsstandards führt zu ähnlichen Überlegungen, die es als Ungerechtigkeit bezeichnen, Frauen von der Teilnahme an prestigereichen wissenschaftlichen Projekten auszuschließen. Obwohl die Sprache der Effizienz anders konnotiert ist als die der Gerechtigkeit, werden beide Begriffe immer wieder benutzt, um die Unterrepräsentanz von Frauen in der Wissenschaft zu kritisieren (vgl. z. B. European Technology 2000). In globalen Fortschritts- und Gerechtigkeitsmodellen wird der gender gap als schwerwiegendes individuelles und gesellschaftliches Problem diskutiert, dessen Bekämpfung wiederum Anlaß zu einer Vielzahl von Konferenzen, wissenschaftlichen Studien und staatlichen Maßnahmen gibt.

Den Ausgangspunkt der Kausalkette bilden zumeist geschlechtsspezifische Unterschiede bei den schulischen Leistungen. Der zweite und der dritte International-Mathematics-and-Science-Studies-Report listen die Ergebnisse von Leistungstests für Mädchen und Jungen in den beteiligten Ländern getrennt auf. Baker, und mit ihm andere Wissenschaftlerinnen und Wissen- 
schaftler, haben diese Daten im Ländervergleich analysiert und aus ihren Ergebnissen lassen sich instruktive Schlußfolgerungen ziehen (Baker/Perkins Jones I993; Baker et al. 2000). Ihre Studien zeigen, daß das Ausmaß der Geschlechterdifferenz bei den schulischen Leistungen im Ländervergleich stark variiert. Diese Ergebnisse laufen biologisch-deterministischen Erklärungen zuwider. Sie zeigen außerdem, daß sich die Kluft zwischen den Geschlechtern verringert und Mädchen zunehmend auch anspruchsvolle Kurse belegen. Insgesamt sprechen diese Befunde für die These, daß egalitäre Geschlechterstandards weit verbreitet sind, und daß diese Verbreitung folgenreich ist. Denn die erhöhte Sensibilität für diese Standards scheint bei Mädchen zu verbesserten Leistungen und zur Belegung von anspruchsvolleren Kursen zu führen. Allerdings bedarf es sehr viel mehr Studien, um die Mechanismen zu bestimmen, durch die diese internationalen Trends entstehen.

Für den ersten und auch den zweiten Bericht stellt Baker fest, daß in den Ländern Mädchen im Vergleich zu Jungen besser abschneiden, in denen Frauen stärker in den relevanten Bildungs- und Berufskategorien vertreten sind. Dies weist darauf hin, daß zur Erklärung der momentanen Leistungsmuster die künftige Gelegenheitsstruktur eine Rolle spielt. Die Wahrscheinlichkeit, daß sich die Leistungen von Mädchen zukünftig in Bildung und Beruf auszahlen, könnte motivierend wirken und eine verstärkte Anstrengung in bestimmten Fächern rechtfertigen. Außerdem werden auch Eltern und Lehrer vermutlich mehr Zeit und Arbeit in die schulischen Leistungen von Mädchen investieren, wenn die zukünftige Bedeutung dieser akademischen Leistungen sich deutlicher abzeichnet. Diese Wahrnehmung verknüpfter Chancen modifiziert die oben skizzierten Kausalketten. Es sind dann nicht nur die heute erbrachten Leistungen, die die Zukunft beeinflussen, sondern auch eine antizipierende Sozialisation (anticipatory socialization) im Hinblick auf zukünftige Möglichkeiten, die sich auf jetzige Aktivitäten und Leistungen auswirkt.

Angesichts dieses Wandels können die verbesserten Zugangschancen von Frauen zum höheren Bildungswesen, die im ersten Teil dieses Beitrags empirisch als weltweiter Trend festgestellt wurden, neu gewichtet werden (vgl. Bradley/Ramirez 1996). Einerseits weisen die verbesserten Zugangschancen im Bildungswesen in Richtung auf ähnliche Veränderungen beim künftigen Zugang zu prestigereichen Berufen. Andererseits sollte der gestiegene Anteil von Frauen im höheren Bildungswesen die geschlechtsspezifischen Leistungsdifferenzen in der Schule verringern. Diese Gerechtigkeits- und Effizienzmaßstäbe sind mit hoher Wahrscheinlichkeit eher in einer Welt anzutreffen, in der Frauen auch Zugang zur Universität haben.

Selbst im Rahmen der liberalen Perspektive wird dieser Optimismus allerdings mit dem Hinweis auf die Heterogenität der verbesserten $\mathrm{Zu}$ - 
gangschancen im höheren Bildungswesen gebremst. D.h., in den prestigeträchtigen Bereichen des Bildungswesens und an den angesehensten Universitäten bleibt die Zuwachsrate für Frauen wahrscheinlich geringer. Angesichts der geschlechtlichen Segregation im höheren Bildungswesen kann man den verbesserten Zugang dann allerdings auch als Phyrrus-Sieg begreifen (vgl. Bradley 2000). Denn eine gründliche Untersuchung der betreffenden Daten zeigt in der Tat, daß Frauen zwar insgesamt einen verbesserten Zugang zum höheren Bildungswesen haben, doch daß dies für die natur- und ingenieurwissenschaftlichen Fächer nicht im gleichen Maße gilt (vgl. Ramirez/Wotipka 200I). Für die Daten von I972, I982 und 1992 bleibt dieser Befund konstant. Es ist tatsächlich schwieriger für Frauen, zu diesen Studienfeldern Zugang zu finden. Gleichzeitig hat aber der Anteil von Frauen in den Natur- und Ingenieurwissenschaften zwischen 1972 und 1992 weltweit zugenommen. Lag der nationale Durchschnitt des Frauenanteils anfangs bei I4 Prozent, so befand er sich 1992 bei 2I Prozent. In einigen Regionen der Welt war der Zuwachs mäßig (Osteuropa), während er sich in anderen sehr deutlich abzeichnete (Asien). Schließlich läßt sich nirgendwo ein Abwärtstrend beobachten. Der Wandel in Richtung Gleichheit der Geschlechter mag zwar nur langsam erfolgen, doch er vollzieht sich in Richtung Egalität.

Doch selbst dieser bescheidene Optimismus kann mit dem Argument untergraben werden, daß es einfacher ist, Verbesserungen im höheren Bildungswesen zu erreichen, sogar in den naturwissenschaftlichen Fächern, als im Beschäftigungssystem (vgl. Jacobs 1996; Charles 1992). Dies zeigt sich bei der Segregation der Berufe und bei den Einkommensunterschieden zwischen Frauen und Männern (aber selbstverständlich auch bei den Unterschieden zwischen Berufen und Tätigkeitsunterschieden). Diese Befunde sollen in der Regel die Fortdauer von Ungleichheit belegen. Allerdings wird dabei zumeist die Tatsache übersehen, daß die Meßlatte für Geschlechtergleichheit angehoben worden ist, und dies Auswirkungen darauf hat, was als relevante Ungleichheit angenommen wird. Um einen entscheidenden Punkt zu wiederholen: Die Anhebung egalitärer Standards erhöht die Wahrscheinlichkeit, daß alte und neue Ungleichheiten entdeckt und als Ungerechtigkeit, aus einigen Perspektiven sogar als Ineffizienz, bezeichnet werden. Aus dieser Sicht scheinen die entdeckten Ungleichheiten nur geringfügige Abstriche bei den eigentlichen Zugewinnen mit sich zu bringen. Dies ergibt sich aus der Forschungsperspektive, nämlich dadurch, daß der Zugang zur Universität statt zum höheren Bildungswesen untersucht wird, oder daß zwischen dem Zugang zum akademischen Lehrkörper und dem zu Studienplätzen im höheren Bildungswesen unterschieden wird. Aber die Entdeckung einiger neuer Ungleichheiten verdankt sich den radikaleren Ansätzen, die den abstrakten, individuellen Staatsbürger in Frage stellen. 
Diese Ansätze plädieren dafür, die gesamte Wissenschaft grundsätzlich neu zu überdenken.

Die zentrale Annahme dieser Ansätze besteht darin, daß die Wissenschaft eine männliche Institution ist. Dies meint selbstverständlich nicht die einfache Feststellung, daß in der Wissenschaft und gerade in hohen wissenschaftlichen Positionen mehr Männer als Frauen vertreten sind. Zwei häufig wiederkehrende Themen der radikalen Positionen sollen im folgenden aufgegriffen werden. Zunächst ist es der grundlegende Gedanke, daß die begriffliche Dichotomie Mann/Frau eine kulturelle Konstruktion darstellt, die dazu verwandt wurde, Frauen sowohl von Männern zu unterscheiden als auch sie ihnen gegenüber abzuwerten. Es handelt sich um eine Dichotomie, die mit anderen primitiven Gegensätzen wie etwa rational/gefühlsbetont ko-variiert. Argumentationen über den männlichen Charakter der Wissenschaft gehen von diesem Konzept der kulturellen Konstruktion der Dichotomie Mann/Frau aus. Im Zuge der historischen Entwicklung der Wissenschaft als Domäne der Rationalität wurde - dieser Argumentation zufolge - die Wissenschaft angeblich durch ihren maskulinen Charakter imprägniert (es ist umstritten, ob dies zufällig oder absichtlich geschah). Diese Verknüpfung garantiert, daß Frauen in der Wissenschaft per definitionem Fremde sind. Eine weitere Überlegung ist, daß das Kriterium von Gleichheit als Ähnlichkeit (sameness) die Wissens- und Existenzformen von Frauen untergräbt. Da die Wissens- und Daseinsweisen von Männern zum relevanten Bezugspunkt werden, verkörpern Frauen das Andere, und sie bezahlen einen Preis dafür, daß sie nicht mit Männern identisch sind. Beide Konzepte tauchen zwar manchmal in demselben Kontext auf, doch sie setzen unterschiedliche Akzente. Aus der ersten Sicht war es eine nach Geschlecht differenzierende und klassifizierende Wirklichkeitswahrnehmung, die zur Vermännlichung der entstehenden modernen Wissenschaft führte und noch heute für Frauen eine Barriere darstellt. Aus dieser Perspektive werden die Unterschiede zwischen den Geschlechtern also durch das Kategoriensystem der Wissenschaft erzeugt und sind durch seine Legitimität und Reichweite bedingt. Die zweite Vorstellung geht davon aus, daß Unterschiede, egal woher sie rühren, entscheidend sind, und daß die Wissenschaft deswegen vermännlicht ist, weil dort nur männliche Orientierungen honoriert werden. Vertreter dieser zweiten Sicht plädieren eher für einen Wandel, der Gleichheit ohne den Zwang zur Ähnlichkeit (sameness) fördert oder zumindest zuläßt. Verfechter des ersten Gedankens befürworten hingegen nicht nur in der Wissenschaft, sondern in der gesamten Gesellschaft, die Abschaffung der geschlechtlichen Klassifizierung von Tätigkeiten und Identitäten.

Ein Ausgangspunkt für die radikale Perspektive könnte folgendermaBen aussehen: Die historische Entwicklung von Wissenschaft als Berufung 
ging Hand in Hand mit der Herausbildung der Mutterschaft und des Hausfrauendaseins als Berufung. Bezogen auf Deutschland mag die Verklärung der Hausfrauenexistenz und der Mutterschaft besonders ausgeprägt gewesen sein, doch diese Modelle wurden auch anderswo institutionell verankert. Die Transformation der Wissenschaft von einer Kuriosität und Freizeitbeschäftigung hin zu den tiefen, idealisierten Bindungen, die mit der Ausübung einer Berufung einhergingen, ko-variierte mit einer Transformation des Familienlebens. Dieses wandelte sich von einem veränderlichen Konglomerat von Erfahrungen, die nicht wissenschaftlich normiert waren, $\mathrm{zu}$ einem vergleichbaren Bereich idealisierter Bindungen, die die Mutterschaft als Berufung priesen. Während durch diese Transformationen im Bereich der Wissenschaft ein unpersönliches Ideal der Rationalität und Objektivität etabliert wurde, entstand im Bereich der Familie ein gefühlsbetontes Ideal von zwischenmenschlichen Beziehungen und von Subjektivität. Die Dichotomien konnten mit der Zeit zunehmend weniger hinterfragt werden, so daß die Bereiche (Wissenschaft und Familie) und die Personen (Männer und Frauen) nur noch mit spezifischen Orientierungen identifiziert wurden. Darüber hinaus erodierte auch die Unterscheidung zwischen Bereichen und Personen, wodurch die bloße Assoziation von Frauen und Wissenschaft ähnlich problematisch wie die von Männern und Kinderpflege wurde. Das Argument lautet nicht, daß Männer und Frauen in besseren Zeiten sowohl in der Kinderpflege als auch in der Wissenschaft gemeinsam tätig waren, sondern daß ihre unterschiedlichen Verhaltensweisen früher nicht vergleichbar rigiden Kategorien und normativen Vorschriften unterworfen wurden. In dem Maße, wie der Wert der Wissenschaft sich erhöht, erhalten auch die damit einhergehenden Orientierungen eine größere Relevanz. Das Resultat ist eine Vermännlichung der Wissenschaft, die für Frauen eine massive Barriere darstellt.

Diese Ausgangsannahmen sind nicht wirklich neu. Sie repräsentieren lediglich eine bestimmte Form der Familiengeschichtsschreibung. Danach war die Ausdifferenzierung der Familie aus der Gesellschaft oder die Entstehung der modernen gefühlsbetonten Familie eine funktionale Anpassung an die Folgen der Industrialisierung, welche wiederum selbst an die Entstehung der modernen Wissenschaft gekoppelt war. Diese Prozesse, die Frauen ausgegrenzt und ihren Beitrag zur Gesellschaft entwertet haben, enthalten aus feministischer Sicht aber kaum etwas spezifisch Funktionales. $\mathrm{Zu}$ wessen Vorteil waren diese Differenzierungsprozesse und durch wessen Arbeit waren sie möglich? So lautete der Protest der feministischen Kritik an den funktionalistischen Varianten dieser Geschichtsschreibung. Wenn man sie von ihren funktionalistischen Annahmen befreit, dann entspricht diese Geschichtsschreibung aber durchaus der Stoßrichtung der radikal-feministischen Kritik an der Wissenschaft als Institution. 
Worin bestehen die Implikationen der radikalen Kritik? I. Frauen in der Wissenschaft sind oftmals gezwungen, ihre Anwesenheit in einem männlich kodierten Bereich zu erklären. 2. Angesichts ihrer zwiespältigen Identität als Wissenschaftlerinnen ist es wahrscheinlich, daß ihre Leistungen weniger Wertschätzung erfahren. In dem Fall, daß ihre außergewöhnlichen Leistungen ihnen als Wissenschaftlerinnen doch Anerkennung verschaffen, wird freilich ihre Identität als Frau in Frage gestellt. Ohne die Koppelung von Rationalität an Männlichkeit wäre dieser Anerkennungsmechnismus nicht plausibel.

Außerdem wird Wissenschaft als Berufung oftmals als eine Ganztagsbeschäftigung imaginiert, die andere ganztägige Verpflichtungen kaum zuläßt und umgekehrt. Somit werden Frauen als potentielle oder tatsächliche Mütter im Wissenschaftsbetrieb an die Peripherie gedrängt. Sicherlich ist dies bei den Pionierinnen der Wissenschaft offensichtlicher gewesen, doch auch heute werden Wissenschaftlerinnen oftmals als token, d.h. als Angehörige einer Minderheit (vgl. Kanther I977) betrachtet. In der Praxis nimmt dies zwei Formen an: Entweder wird die Frau als Ausnahme-Erscheinung integriert, die letztlich die Regel des Du-bist-wirklich-eine-von-uns bestätigt, oder aber sie muß zu allen möglichen Angelegenheiten die weibliche Sicht der Dinge beisteuern. Beide Dynamiken stärken das Wir, jene männliche Sichtweise, die die Norm darstellt. Es ist ein schwieriges Unterfangen, diese Dynamik nicht zu akzeptieren, und es endet oft mit dem Austritt aus der Wissenschaft. Aus liberaler Perspektive stellt die verstärkte Repräsentanz von Frauen in den Institutionen den Schlüssel zum Erfolg dar (vgl. ebd.). Aus radikaler Perspektive bildet die Normalität der Männlichkeit in der Wissenschaft hingegen das zentrale Problem, und folglich kann die Lösung auch nicht darin bestehen, mehr Frauen die Chance zu geben, männliche Wissenschaftler zu werden.

Die Konzentration auf Lehrpläne und Ausbildung betreffen weitere Implikationen dieser Kritik. Auch hier geht es nicht darum, daß Frauen einen besseren Zugang zu anspruchsvollen Lehrplänen und Lehrern an Schulen und Universitäten erhalten sollten. Die Kursauswahl, die Belegung der Hauptfächer, die Mitgliedschaft in wissenschaftlichen Forschungsteams und angesehenen Labors ist vor allem ein liberales Anliegen. Aus radikaler Perspektive wird dagegen thematisiert, warum einige Kurse oder Studienfächer höher bewertet werden als andere, weil angenommen wird, daß die höher bewerteten Fächer vor allem Männer anziehen. Ein weiterer wichtiger Untersuchungsgegenstand ist die Frage, wie Wissen vermittelt wird, und welche Ausbildung Lehrkräfte für einen effektiven Unterricht erhalten. Der zentrale Gesichtspunkt in der betreffenden Literatur ist, daß Mathematik und Naturwissenschaften in einer Weise unterrichtet werden können, die Mädchen stärker einbindet. Aus dieser Perspektive wird daher zumeist das 
verteidigt, was als reformierte Mathematik oder kontextualisierte und integrierte Naturwissenschaft bekannt ist, weil diese Konzepte Mädchen angeblich mehr entgegenkommen (vgl. Boaler 1997). Herkömmliche Ansätze, Wissen aufzuspalten und zu vermitteln, werden demgegenüber als mädchenfeindlich kritisiert.

In der Praxis schließen sich liberale Feministinnen selbstverständlich jeder Kritik an Lehrplänen und Lehrformen an, die im Verdacht stehen, Mädchen und Frauen zu benachteiligen. Ebenso selbstverständlich setzen sich radikale Feministinnen für die verstärkte Einbeziehung von Frauen in die Wissenschaft ein. Doch sie hinterfragen eher die Bedingungen der Inklusion und befassen sich mit dem Problem der Kooptierung, während die liberalen Positionen dazu neigen, eine Korrelation zwischen stärkerer Inkorporation und Machtzuwachs anzunehmen. Das Ziel der stärkeren Inklusion von Frauen unter veränderten Bedingungen bildet die Gemeinsamkeit beider Positionen. Die politische Maßnahmen, die mehr Frauen in die Wissenschaft bringen und diese zugleich entmännlichen, werden bevorzugt. Reformversuche schließen alltägliche Praktiken wie die Produktion von Textbüchern und Lehrplänen ein, die Wissenschaft nicht nur als von Männern produziertes Wissensfeld präsentieren, das in typisch männlichen Kontexten entsteht. Dieses politische Projekt umfaßt auch tiefergreifende Heraus forderungen wie die Darstellung von Wissenschaft als Kooperation zwischen Gemeinschaften von Forschern, statt als Wettbewerb zwischen charismatischen Einzelpersonen.

Der Eintritt von Frauen in die Wissenschaft mag, wie es die liberalen Feministinnen behaupten, ihren maskulinen Charakter vielleicht nicht direkt verändern. Doch ihre Zunahme ist nicht folgenlos. In einer Welt, in der individuelle Rechte vielleicht bald auch das Recht auf eine kollektive und vielfältige Identität beinhalten, wie etwa Geschlecht und Ethnizität, kann die Grenze zwischen individuellen und kollektiven Rechten erodieren. Dies könnte wiederum die Unterscheidung zwischen liberalen und radikalen feministischen Positionen verwischen. Der ironische, vielleicht unbeabsichtigte und indirekteste Effekt dessen könnte darin bestehen, daß die steigende Anzahl von Frauen in der Wissenschaft zu ihrer Entmaskulinisierung führt und damit auch das Verhältnis zwischen Frauen und Wissenschaft verändert.

\section{Schlußbemerkung}

Die Agenda des liberalen Feminismus besitzt eine deutliche Affinität zu globalen Fortschritts- und Gerechtigkeitsmodellen und wird durch diese verstärkt. Angesichts der wachsenden Bedeutung der Wissenschaft als Ent- 
wicklungsmodell ist es nicht überraschend, daß liberale feministische Kritiken in ihr fest verankert sind. Denn beide feministische Kritiken basieren auf der Wahrnehmung erweiterter egalitärer Standards und dehnen diese aus. Politische Planer, für die umfassendere Gleichheitsstandards selbstverständlich sind, beschäftigen sich mit weiteren Ungleichheiten zwischen den Geschlechtern. Der Informationsbedarf vieler dieser Planer, die Frauen und Männer in den Wissenschaftsbereichen vergleichen, ist direkt auf das Estab. lishment liberaler feministischer Kritik zurückzuführen. Diese Kritik fokussiert auf Frauen als Individuen und betont ihr Humankapital-Potential sowie ihre staatsbürgerlichen Rechte und Menschenrechte. Aus der Perspektive der Menschenrechte und des Humankapital-Ansatzes verbessern mehr Frauen in der Wissenschaft die Situation von Frauen als Individuen und auch die der Gesellschaften.

Da sie die Bedingungen der Inklusion von Frauen in die Wissenschaft direkter hinterfragen, sind radikale feministische Ansätze problematischer. Sie fordern eine Entmaskulinisierung der Wissenschaft. Hierfür ist wiederum zweierlei notwendig, nämlich die Koppelung von Rationalität und Männlichkeit aufzulösen und ein Gleichheitskonzept ohne die Norm der Ännlichkeit (sameness) anzustreben. Es reicht nicht, daß mehr Frauen so werden können wie männliche Wissenschaftler sind. Der radikale feministische Diskurs konzentriert sich daher auf Frauen und Wissenschaft, nicht nur auf Frauen in der Wissenschaft.

Ein großer Teil der Literatur zu Frauen in der Wissenschaft/Frauen und Wissenschaft hat keinen Bezug zur makrosoziologischen Forschung über den weltweiten Wandel von staatsbürgerlichen Rechten und Menschenrechten von Frauen. In diesem Forschungsbereich wird indes weltweit ein Trend hin zu mehr Rechten für Frauen konstatiert. Nationale sowie internationale Bürokratien und Verbände beschäftigen sich mit diesen Entwicklungstrends und deren Grenzen. Ihre Debatten haben sich im Laufe der Zeit verändert: Lag der Schwerpunkt früher auf Rechten, die zunächst nur Männer erhielten und dann von Frauen eingefordert wurden, so geht es inzwischen um Frauenrechte, die Frauen als Frauen erwerben. In diesen Debatten entstehen neue Rechte und neuartige Diskussionen über Formen kultureller Vielfalt. In einer sich verändernden Welt, in der kollektive Identitäten möglicherweise individuelle Rechte werden, könnte sich auch das jetzige Verständnis von liberalen und radikalen Perspektiven auf Frauen in der Wissenschaft verändern. 


\section{Literatur}

Anderson, Benedict (I99I): Imagined Communities: Reflections on the Origin and Spread of Nationalism, New York/NY: Verso.

Baker, David P./Perkins Jones, Deborah (I993): „Creating Gender Equality: Cross-national Gender Stratification and Mathematical Performance», in: Sociology of Education 66, S. 9I-I03.

Baker, David P./Riegle-Crumb, Catherine/Wiseman, Alex W./LeTendre, Gerald K./Ramirez, Francisco O. (2000): Shifting Gender Effects: Opportunity Structures, Mass Education, and Cross-national Achievement in Mathematics and Science, Pennsylvania State University, School of Education, unveröffentlichtes Manuskript.

Bendix, Reinhard (1964): Nation-Building and Citizenship: Studies of Our Changing Social Order, Berkeley/CA: University of California Press.

Berkovitch, Nitza (I999): From Motherhood to Citizenship: Women's Rights and International Organizations, Baltimore/MD: Johns Hopkins University Press.

Boaler, Jo (I997): "Reclaiming School Mathematics: The Girls Fight Back«, in: Gender and Education 9, S. 285-305.

Boli, John (1987): "Human Rights or State Expansion? Cross-National Definitions of Constitutional Rights, I870-1970«, in: George M. Thomas/ John W. Meyer/Francisco O. Ramirez/John Boli (Hg.), Institutional Structure: Constituting State, Society, and the Individual, Newbury Park/ CA: Sage, S. 133-I49.

Boli, John/Thomas, George M. (I997): »World Culture in the World Polity: A Century of International Non-Governmental Organization«, in: American Sociological Review 62, S. I7I-I90.

Bradley, Karen (2000): "The Incorporation of Women into Higher Education: Paradoxical Outcomes«, in: Sociology of Education 73, S. I-I8.

Bradley, Karen/Ramirez, Francisco O. (I996): „World Polity and Gender Parity: Women's Share of Higher Education, 1965-1985«, in: Aaron M. Pallas (Hg.), Research in Sociology of Education and Socialization II, Greenwich/CT: JAI, S. 63-9I.

Chabbott, Colette (2002): Constructing Educational Development: International Development Organizations and the World Conference on Education for All, New York/NY: Falmer Press.

Charles, Maria (1992): "Cross National Variation in Occupational Sex Segregation«, in: American Sociological Review 57, S. 483-501.

Drori, Gili S. (1997): The National Science Agenda as a Ritual of Modern Nation-Statehood: The Consequences of National >Science for National Developmente Projects, Stanford/CA: Stanford University, unveröffentlichte Doktorarbeit. 
Drori, Gili S./Meyer, John W./Ramirez, Francisco O./Schofer, Evan (2002): Science in the Modern World Polity: Institutionalization and Globalization, Stanford/CA: Stanford University Press.

European Technology Assessment Network (ETAN) on Women and Science (2000): Science Policies in the European Union: Promoting Excellence Through Mainstreaming Gender Equality, Brüssel: Europäische Kommission.

Hanson, Sandra L./Schaub, Maryellen/Baker, David P. (I996): „Gender Stratification in the Science Pipeline: A Comparative Analysis of Seven Countries«, in: Gender \& Society 10, S. 271-290.

Jacobs, Jerry A. (I996): „Gender Inequality and Higher Education«, in: Annual Review of Sociology 22, S. 153-185.

Kanther, Rosabeth Moss (I977): "Some Effects of Proportions in Groups Life: Skewed Sex Ratios and Responses to Token Women«, in: American Journal of Sociology 82, S. 965-990.

Marshall, Thomas H. (1964): Class, Citizenship, and Social Development, Garden City/NY: Doubleday.

McEneaney, Elizabeth H./Meyer, John W. (I999): »The Content of the Curriculum: An Institutional Perspective «, in: Maureen Hallinan (Hg.), Handbook of Sociology of Education, New York/NY: Plenum, S. I89-2I2.

Meyer, John W./Boli, John/Thomas, George M./Ramirez, Francisco O. (1997): "World Society and the Nation-State«, in: American Journal of Sociology I03, S. 144-I8I.

O'Connor, Julia S./Orloff, Ann Shola/Shaver, Sheila (I999): States, Markets, Families: Gender, Liberalism, and Social Policy in Australia, Canada, Great Britain, and the United States, New York/NY: Cambridge University Press.

Okin, Susan (I989): Justice, Gender and the Family, New York/NY: Basic Books.

Orloff, Ann Shola (1993): »Gender and the Social Rights of Citizenship: The Comparative Analysis of Gender Relations and Welfare States «, in: American Sociological Review 58, S. 303-328.

Pateman, Carole (I995): Participation and Democratic Theory, Cambridge: Cambridge University Press.

Pateman, Carole (I988): The Sexual Contract, Stanford/CA: Stanford University Press.

Ramirez, Francisco O./Cha, Yun-Kyung (I990): "Citizenship and Gender: Western Educational Developments in Comparative Perspective «, Research in Sociology of Education and Socialization 9, S. I53-I73.

Ramirez, Francisco O./McEneaney, Elizabeth H. (I997): »From Women's Suffrage to Reproduction Rights? Cross-National Considerations«, in: International Journal of Comparative Sociology 38, S. 6-24. 
Ramirez, Francisco O./Soysal, Yasemin/Shanahan, Suzanne (1997): „The Changing Logic of Political Citizenship: Cross-National Acquisition of Women's Suffrage Rights, I890 to I990«, in: American Sociological Review 62, S. 735-745.

Ramirez, Francisco O./Wotipka, Christine Min (200I): »Slowly But Surely? The Global Expansion of Women's Participation in Science and Engineering Fields of Study, 1972-92«, in: Sociology of Education 74, S. 23I251.

Schofer, Evan/Ramirez, Francisco O./Meyer, John W. (2000): »The Effects of Science on National Economic Development, 1970-1990«, in: American Sociological Review 65, S. 866-887.

Tiberghien, Jennifer (1997): The Power of Unarmed Prophets: Non-Governmental Organizations as Carriers of Human Rights Organizations, Stanford University, School of Education: Masters Thesis.

Tiberghien, Jennifer (2000): »Daring to Talk: Nongovernmental Organizations and the Diffusion of Norms on Violence Against Women in Japan.« Vortrag, Jahrestreffen der National Women's Studies Association, Boston/MA. 